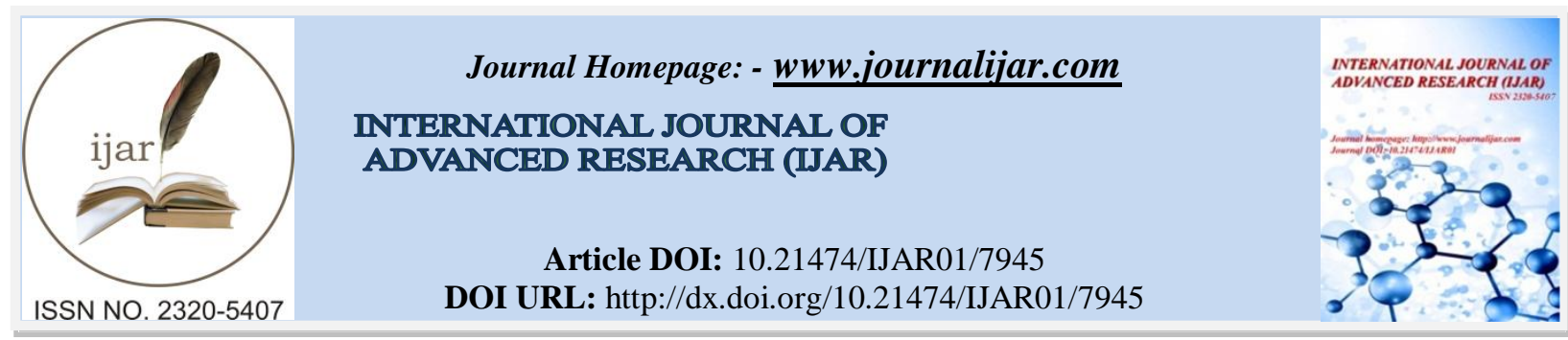

RESEARCH ARTICLE

\title{
MIELEKEO YA WANAFUNZI WA KIDATO CHA TATU NA CHA NNE KUHUSU METHALI KINZANI: UCHUNGUZI KATIKA SHULE ZA UPILI KATIKA KATA YA TOWNSHIP, KAUNTI YA KITUI; KENYA.
}

\author{
Mbusya Mutei Catherine \\ Dr. Chomba Esther.
}

\section{Manuscript Info}

\section{Manuscript History}

Received: 16 August 2018

Final Accepted: 18 September 2018

Published: October 2018

Keywords:- Ukinzani, Methali za Kiswahili, mtumiaji lugha, Jozi za methali, Mwelekeo

\section{Abstract}

Ukinzani ni dhana ambayo imefasiliwa kama ubishi au upinzani. Ukinzani katika methali za Kiswahili unaweza kufasiliwa kama unyume wa kimaana unaoletwa na methali ambazo ziko katika jozi moja na zinazotoa maana zilizo kinyume. Methali za Kiswahili zina maana na ili maana hiyo ijitokeze kwa namna inayofaa, kila methali inapaswa kutumika katika mazingira yanayooana na maana ya methali mahususi. Methali zina jukumu la kuonya, kusifu, kukashifu, kuelekeza, kuliwaza na kadhalika. Aidha, katika fasihi ya Kiswahili kuna jozi za methali zilizo na maana kinyume. Kwamba, methali moja inakuelekeza huku na nyingine inakupa mwelekeo unaotofautina na ule wa awali. Katika hali kama hii, mtumiaji lugha na mwanajamii kwa jumla anaweza kupatwa na utata wa mwelekeo atakaoufuata. Methali zinapotoa maana zinazopingana basi utata unaweza kujitokeza haswa ukimsemea nwanajamii methali kisha naye akutajia iliyo na maana kinyume, huenda suluhu ya mnachozungumzia ikakosekana. Hali hii ndiyo ilimsukuma mtafiti akitaka kubainisha namna wanajamii wanavyochukulia kuwepo kwa ubishi katika methali za Kiswahili, ikizingatiwa kwamba methali hizi hutumika kila mara na wanajamii katika mazungumzo, nyimbo na hata katika uandishi.

Copy Right, IJAR, 2018,. All rights reserved.

\section{Utangulizi:-}

Neno ukinzani limefasiliwa na Kamusi ya Kiswahili sanifu (2004) kama hali ya ukaidi na kisawe chake ni upinzani. Upinzani ni hali ya kupinga au kuwa na ubishi kuhusu suala fulani. Hali hii hutokea katika mazingira ya kutokuwepo na mwafaka kuhusu suala au hali fulani inayojadiliwa au kuzungumziwa.

Tunapozingatia methali za Kiswahili tutapata ubishi hujitokeza wakati ambapo maana za methali zinapingana au zinatoa maana zilizo kinyume. Kila methali ya Kiswahili huwa na maana yake lakini katika mazingira ambayo methali mbili huwa na maana zilizo kinyume kuhusu suala moja, huu ndio hufahamika kama ukinzani wa methali. Akutendaye ubaya mlipe kwa wema/ Akutendaye mtende ni mfano wa methali zilizo na ukinzani ulio wazi. Methali ya kwanza inashawishi wanajamii kutolipiza kisasi huku ya pili ikitoa shinikizo lililo kinyume kabisa; inasisitizia kisasi miongoni mwa wanajamii. 
Methali ni semi ambazo hutumiwa na watumiaji lugha kuondoa ukungu katika mawasiliano ambayo yanaonekana kuwa magumu ama yaliyo na usayansi. Mwandishi Chinua Achebe (1959) ananukuliwa na John. P (2005) akisisitiza kwamba methali ni mafuta ya mawese ambayo kwayo maneno huliwa. Kwa kuzingatia kauli hii, lugha ya fasihi hutumia methali na fani ziginenezo kama viungo vya kuifanya lugha hii iwe na ladha ya kupendeza miongoni mwa watumiaji wake.

Methali zinalinganishwa na chakula ambapo ili chakula kipate ladha ya kupendeza lazima kitiwe viungo tofauti na vichanganywe kwa utaratibu mzuri na wa kupendeza. Vivyo hivyo, methali zinapotumiwa lazima mtumiaji lugha atumie methali mwafaka kwa muktadha mahususi ili kuleta maana katika mawasiliano. Haiwezekani kuchukua methali yoyote tu na 'kuitupa' popote pale katika mawasiliano. Methali hiyo itakosa maana kwani haioani na muktadha mahususi.

Methali ni kipera cha utanzu wa semi katika fasihi simulizi. Kulingana na TUKI (2004) methali ni usemi wa kisanii ambao ni wa kimapokeo na jamii huuchukulia kuwa wa kweli na hutumiwa na jamii husika kufumbia au kupigia mfano. Vilevile, methali hubeba maana pana kuliko maneno yanayotumiwa. Kwa mfano, tunaposema kwamba dawa ya meno ni meno, neno meno limebeba maana zaidi ya kile kipande kigumu na cheupe kilicho kinywani mwa binadamu au mnyama.

Kwa mujibu wa Wamitila (2003), methali inafasiliwa kama msemo wa kimapokeo na ambao muundo wake ni maalum, unaweza kukumbukika na malengo yake ni kama vile kuadibu, kulaumu, kushauri, na kadhalika. Suala la methali limeshughulikiwa sana katika Paremiolojia. Methali huwa na miundo tofauti tofauti kwa mfano, zipo methali ambazo zimeundwa kwa kutumia maswali ya balagha. Kwa mfano, kamba haikuwa kamba, itakuwa kambaa?

Utafiti kuhusu methali umefanywa na watafiti mbalimbali ambao wameshughulikia taaluma hii ambayo ni pana na changamano. Kadiri wachunguzi wanavyozidi kushughulika na kazi yao ya utafiti ndivyo wanavyozidi kufichua maarifa muhimu na kuyatumia katika nyanja za masomo ya juu ulimwenguni. Hata hivyo, ni wazi kwamba hiki kipera cha methali hakijatafitiwa vya kutosha.

Tofauti na tafiti zilizofanywa hapo awali, utafiti huu uliangazia ukinzani katika methali za Kiswahili. Katika uwanja huu mpana wa methali za Kiswahili, ni wazi kwamba zipo methali ambazo hupinga maana ya methali nyingine, hivi kwamba methali moja inatoa ujumbe fulani huku ya pili ikitoa ujumbe unaoonyesha kinyume cha ujumbe uliotangulia. Kwa mfano:

1. Mavi ya kale hayanuki

2. Mavi ya kale hayaachi kunuka.

Kauli hii ina maana kwamba kila jamii ina methali zake ambazo jamii mahususi huzitumia mara kwa mara katika kuwasilisha mawazo tofauti tofauti. Methali zinazohusishwa na jamii fulani, huwa amali na sehemu ya utamaduni wa jamii husika. Hata hivyo, kuna methali ambazo hutumika mahali popote duniani. Kwa mujibu wa John C.N (2011) anasema kwamba kuna methali zilizo na uwezo wa kuvuka mipaka na kutumika kwingine ambako lugha yake ni tofauti. Kinachovuka mipaka sio muundo wa methali bali ni maudhui au maana inayotolewa na methali hiyo. Yaani, maneno tofauti hutumika lakini maana huwa sawa. John (2005) ameziita methali hizi methali za kiutandawazi au methali za kimataifa. Kwa mfano, akili ni nywele, kila mtu ana zake na tamaa mbele mauti nyuma.

Katika utafiti huu, suala lililodhamiriwa kutafutiwa ufumbuzi ni kubaini mielekeo ya watumiaji lugha ya Kiswahili kuhusu suala la ukinzani wa methali. Uchunguzi ulifanywa kwa wanafunzi wa fasihi katika kiwango cha shule ya upili, kidato cha tatu na cha nne. Hii ni sampuli tosha katika utafiti huu kwani ni watumiaji wa lugha ya Kiswahili na wanaingiliana na methali za Kiswahili kila mara; katika mawasiliano na uandishi. Kwa mujibu wa Mtesigwa (2013), maana ya methali hutofautiana miongoni mwa watumiaji hata ingawa watumiaji hawa wamewahi kuisikia ikitumiwa. Kutokana na utafiti wake, inabainika kwamba ugumu wa kutambua maana na matumizi ya methali hutegemea dhamira ya msemaji na mazingira yake ya matumizi. 
Methali zina jukumu la kuleta suluhu ya baadhi ya matatizo miongoni mwa watumiaji lugha. Je, methali zinapokinzana huleta suluhisho kama inavyotarajiwa? Maswali huibuka kuhusu methali itakayofaa kutumiwa wakati huo mahususi ili kutoa suluhisho mwafaka. Kwa mfano:

1. Nahodha wengi chombo huenda mrama

2. Panapo wengi hapaharibiki jambo.

Katika hali kama hii, upande wowote ule unaweza ukajitetea kwani methali hizi zinakinzana kimaana. Upande mmoja utakashifu ule mwingine kwa kuhusisha watu wengi katika kutatua jambo fulani. Kwa upande, mwingine hao wengi watajitetea kwa kuwapingia methali, panapo wengi hapaharibiki jambo. Hali hii ndiyo iliyomsukuma mtafiti kufanya utafiti huu lengo likiwa kubaini mielekeo mahususi inayodhihirika kutoka kwa watumiaji lugha, kutokana na ubishi unaoletwa na methali zilizo na maana kinyume. Athari hii inaweza kuwa chanya au hasi kutegemea mtumia lugha mmoja hadi mwingine.Mtafiti alitumia wanafunzi na walimu wa fasihi ya Kiswahili kama sampuli tosha ili kufanikisha utafiti huu. Maoni waliyotoa wanafunzi na walimu yaliongoza katika kufikia malengo ya utafiti huu.

Utafiti huu ulilenga kubaini mielekeo ya wanafunzi na walimu katika kiwango cha shule ya upili wakiongozwa na malengo yafuatayo:

1. Kubainisha mielekeo ya wanafunzi wa kidato cha tatu na cha nne kuhusu methali zenye maana kinzani.

2. Kutathmini mielekeo ya wanafunzi wa kidato cha tatu na cha nne kwa kutumia kigezo cha uchanya na uhasi.

I.

\section{Udurusu wa Maandishi}

Kuna mengi yaliyoandikwa kuhusu methali kama utanzu wa fasihi simulizi lakini suala la ukinzani katika methali halijashughulikiwa vya kutosha haswa katika lugha ya Kiswahili. Utafiti huu utashughulikia kwa kina athari zinazoletwa na ukinzani wa methali za Kiswahili kwa watumiaji wa lugha hii. Kwa mujibu wa Yankah (1994), wanadamu wana uelewa mkubwa wa kuwepo kwa methali kinzani katika lugha. Anatoa mifano ya jozi ya methali ambayo ni kinzani katika lugha ya Kiingereza;

"Absence makes the heart go fonder" hii ni methali ambayo ilichapishwa mara ya kwanza katika kitabu cha mashairi cha Francis Davison's Poetical Rhapsody mwaka wa 1602 na ile ya "Out of sight out of mind" ambayo tafsiri yake katika lugha ya Kiswahili ni 'Asiyekuwepo na lake halipo'. Hata hivyo, anasisitiza kuwa ukinzani huu haupaswi kuwa jambo la ajabu sana kwani methali ni semi za hekima ambazo hutumiwa tu katika mazingira mahususi na jamii au watu fulani. Kinachotiliwa mkazo na kauli hii ni kwamba methali zikitumiwa katika muktadha na matumizi yanayofaa, basi ukinzani huwa haupatikani. Methali hupata maana yake ikitumika katika muktadha unaofaa.

Kawaida, methali hujengwa na mazingira kwani misamiati inayopatikana katika methali inaoana na mazingira fulani. Hii ndio sababu tutashuhudia baadhi ya methali ambazo zinapatikana na kutumiwa tu na wanajamii mahususi. Fasili nyingi za fasihi huwa zimeegemea kuielezea katika uhusiano wake na jamii. Fasili hizi nizadhihirisha wazi kwamba fasihi ni taasisi ya jamii na uhusiano wake na jamii hauwezi kupuuzwa. Escarpit, $\mathrm{R}$ (1974; 4) anaunga mkono maoni haya kwa kusisitiza kwamba lazima fasihi ichukuliwe kama iliyo na uhusiano usiotatanika na uhusiano wa kijamii. Fasihi huwakilisha maisha na maisha haya kwa upana huonyesha uhalisia wa jamii.

Methali bado zina maana. Nzookwa J. (2002) anasema kwamba methali fulani hutolewa kama mukhtasari wa uzoefu alionao mtu katika jambo fulani au jamii mahususi. Jamii husikika ikitumia methali kila uchao bila kuzingatia iwapo ni ya zamani au ya sasa. Methali mpya huzidi kuundwa kulingana na jamii inavyozidi kukabiliana na matukio tofauti katika maisha yao. Iwapo binadamu fulani hana uzoefu wa kutosha katika shughuli fulani, itakuwa vigumu kupata methali ya kutumia kama mukhtasari wa kauli aliotaka kuitoa. Methali inapaswa kutoa ujumbe fulani na lazima mtu huyu atoe methali inayofaa muktadha husika.

Mieder na Litovkina (1999) na Mieder (2007) wanasisitiza kwamba methali huzaliwa kila siku. Kulingana nao, methali huwa muundo wa; mada na maelezo ya mada hiyo. Methali huwa na sehemu mbili ambapo sehemu ya kwanza hutoa hoja huku sehemu ya pili ikijibu au kufafanua sehemu huu ya kwanza. Kwa mfano, 'asiyefunzwa na mamaye, hufunzwa na ulimwengu'. 
Kuhusiana na suala la ukinzani katika methali, waandishi hawa wanasema kwamba katika siku za hivi karibuni watumiaji lugha wanazipinda methali za Kiswahili ili kuleta hali dhihaka lakini iliyo na ukweli. Wanatoa mfano wa mwandishi Mochiwa (1988) ambaye alipinda methali isemayo 'mvumilivu hula mbivu' ikawa 'mvumilivu hula mbovu'. Katika uwanda mkubwa wa methali kuna mazingira ya methali kongwe, methali pindwa na methali ibukizi. Hizi ni tu njia tofauti za kufikisha ujumbe ulio ndani ya methali.

Methali huundwa kwa sababu kadhaa na kwa njia tofauti. Nsookwa anasema kwamba zipo methali ambazo hutokea kama kauli ya kawaida na hatimaye kupandishwa hadhi ya methali. Ni wazi kwamba methali hutumiwa mara kwa mara kutegemea muktadha fulani. Zipo methali za Kiswahili ambazo zinatofautiana kwa namna fulani lakini huonyesha uhusiano wa matumizi yake. Kwa mfano, huwezi kumwashia jirani taa na hali kwako kuna giza. Methali hii inahusiana kimaana na ile inasema kwamba, usiache kwako kumezimika ukaenda kuwasha kwa jirani. Vilevile, kuna methali nyingi za Kiswahili ambazo zinatoa ujumbe kinyume. Hili ndilo suala ambalo utafiti huu utajikita kwalo.

Katika karne ya kwanza, mwandishi wa Kirumi aitwaye Lucretius alitoa msemo ambao katika mwaka wa 1604 ulifanywa kuwa msemo katika lugha ya Kiingereza; 'One man's meat is another man's poison' tafsiri ya methali hii katika lugha ya Kiswahili ni 'Mkuki kwa nguruwe mtamu, kwa binadamu uchungu.' Methali hii ina maana kwamba unachokipenda huenda kikiwa chukizo kwa mtu mwingine. Katika maisha ya kawaida, watu huwa na hisia tofauti kuhusu jambo au hali fulani. Ujumbe huu hutumiwa kumsuta mtu ambaye hupenda kuwahujumu wengine lakini wakati wake wa kuhujumiwa ukifika atalalamika kwamba anaonewa.

Hata hivyo katika mwaka wa 1546, John Heywood alikuwa keshatoa msemo unaokinzana na ule wa Lucretius usemao, 'What good for the goose is good for the gander'. Ikiwa na maana kwamba kilicho kizuri kwako hata kwa mwingine ni vivyo hivyo.

Kwa kufafanua methali hizo mbili nilizotaja hapo juu: Absence makes the heart go fonder inatumiwa sana na watu wanaopendana na ina maana kwamba kadri mmoja anavyokuwa mbali na mwenzake, mapenzi nayo yanazidi kunoga. Methali hii ina maana kinyume na inayosema kwamba 'kisicho machoni na moyoni hakipo pia'.

Kwa upande mwingine, "Out of sight out of mind", inaleta ukinzani ufuatao, kwamba kisicho machoni na moyoni hakipo pia na bila shaka kitasahaulika. Kwa mfano, mtu akienda kuisha mbali na mpenziwe, mara nyingi mapenzi kati yao hupunguza na hata wengine kutengana kabisa. Utengano huu husabishwa na umbali huo. Ukinzani katika methali hizi mbili utapatikana iwapo kwa mfano, aliyezoeana na mpenziwe ama rafikiye wa karibu watatenganishwa. Katika hali hii ya upweke methali 'absence makes the heart go fonder' huenda itakosa maana mbele ya upeo wa mtu huyu. Je, wahenga hawakusema kwamba 'fimbo ya mbali haini nyoka'?

Yankah anazidi kufafanua suala hili la ukinzani wa methali na kusema kwamba, iwapo mtu atachukulia miwani kama jambo au hali halisi katika jamii fulani, basi ukinzani hautakosekana katika methali zinazopatikana katika jamii hiyo. Hivyo basi anatoa maoni kwamba iwapo methali itatumiwa katika na kulingana na mazingira mahususi na yanayofaa, basi ukinzani katika methali hautapatikana [utafsiri]. Kawaida, methali hujengwa na mazingira kwani misamiati inayopatikana katika methali inaoana na mazingira fulani. Hii ndio sababu tutashuhudia baadhi ya methali ambazo zinapatikana na kutumiwa tu na wanajamii mahususi. Kwa mfano, “mgaagaa na upwa hali wali mkavu” hii ni methali ambayo iwapo tutazingatia maana ya nje, watakayoielewa vyema na haraka ni wale tu ambao wanapatikana katika maeneo ambayo misamiati kama vile upwa na wali inatumika.

\section{Msingi wa Nadharia}

Kinadharia, utafiti huu uliongozwa na nadharia mbili; ya mguso na ya umaanishaji. Nadharia ya mguso inasisitiza kwamba lazima fasihi iwe inamlenga mtu au watu fulani kwa njia moja ama nyingine ili kuwafanya waifikirie fasihi hiyo kwa undani wa aina yake. Kwa upande wa pili, nadharia ya umaanishaji nayo husisitiza maana katika mawasiliano, ambapo lazima wanaohusika waelewe maana inayojitokeza katika fasihi husika.

\section{Nadharia wa Mguso}

Nadharia ya mguso inafafanuliwa kwa kina na Ntarangwi (2004:14) ambapo anaihusisha na jamii. Anasema kwamba, kila kazi ya fasihi huwa inamlenga mtu au kundi la watu fulani kwa namna kwamba lazima itoe mguso fulani kwa hadhira hiyo. Hadhira basi itafikiria zaidi na hata kujiuliza maswali kuhusu fasihi hiyo na basi kupata 
funzo fulani kutokana na fasihi hiyo. Ntarangwi anaunga mkono maoni ya Vasquez A.S (1973; 113) ambaye anasema kwamba:

...kazi ya sanaa huathiri watu na inachangia katika kuhimiza au kupuuza dhana zao, maazimio yao hata aadili yao-ina msukumo wa kijamii ambao huathiri watu kwa nguvu zake za kihisia na kiitikadi. Ama kwa hakika, hakuna anayebaki vile vile baada ya kuguswa na kazi halisi ya sanaa. [Tafsiri yetu]

Kwa mujibu wa Vasquez A.S basi, lazima fasihi iwe na athari fulani katika maisha ya binadamu husika na mguso huu utampa msukumo fulani ambao utamwelekeza katika kuathirika kihihisia au hata kitabia. Anasisitiza kwamba unapoguswa na fasihi huwezi ukabaki kama ulivyokuwa hapo awali. Kwa kutolea hoja hii mfano, unapokuwa katika hali ya wasiwasi kwamba hufikii malengo yako maishani kisha uambiwe methali kwamba, "Kupotea njia ndiko kujua njia." Methali hii itakuhimiza kwamba usichoke kujaribu kwani siku moja utafanikiwa. Hivi ni kumaanisha kwamba itakugusa kihisia na kukuhimiza usonge mbele.

Kwa ujumla, nadharia hii inashikilia kwamba kazi ya fasihi lazima iwe inalenga mtu au kundi fulani la watu. Methali kama utanzu wa fasihi zinapaswa kuleta mguso fulani kwa watumiaji lugha. Fasihi ina msukumo wa kijamii unaochangia katika kuathiri jamii husikika kitabia, kimsimamo na kiitikadi. Kauli hii inasisitiza kwamba fasihi huleta badiliko fulani katika maisha ya mwanajamii. Wellek na Warren (1949:102) wanashuhudia kuwa watu wamewahi kubadilisha mienendo yao katika maisha kwa kuathiriwa na wahusika wa kubuni katika fasihi.

Katika utafiti huu, maoni ya wanafunzi na walimu wa fasihi yalisaidia katika kutathmini mguso unaoletwa na methali kinzani. Kwa hakika mielekeo yao kuhusu suala hili linalotafutiwa, ni mojawapo wa mguso unaoletwa na ukinzani katika methali. Athari anayoipata mwanajamii mahususi kutokana na ukinzani wa methali, iliongoza katika kutosheleza malengo ya utafiti huu. Mtafiti aliongozwa na maoni ya kila mtafitiwa katika kubainisha mwelekeo wake kuhusu ukinzani wa methali na baadaye kubaini iwapo mwelekeo huu ni chanya au ni mwelekeo hasi. Nadharia hii inasisitizia kuwepo kwa mguso fulani kutokana na kazi ya fasihi. Mhimili huu uliongoza utafiti huu katika kubaini mielekeo ya wanafunzi kutokana na mguso au athari wanayopata wanapokabiliana na suala la ukinzani wa methali.

Ingawa nadharia hii ya mguso ina changamoto ya kutathmini kiwango cha mguso, katika utafiti huu nina matumaini kwamba data iliyokusanywa ilisaidia katika kukabiliana na changamoto hii.

\section{Nadharia ya Umaanishaji}

Umaanishaji ni neno ambalo msingi wake unahusiana na dhana ya 'maana'. Nadharia hii a umaanishaji iliasisiwa na Grice (1975). Mhimili wake wa msingi kabisa ni kwamba katika fasihi, mambo huwa hayasemwi waziwazi. Ili kuelewa kinachosemwa lazima pawepo na umaanishaji katika maana.

Wasemaji huweza kumaanisha mambo yanayozidi wanachokisema. Kauli hii ina maana kwamba fasihi hutumia lugha, ambapo neno moja linaweza kuwa maana zaidi ya ile ya msingi. Hivyo basi, lazima msikilizaji aelewe na kuchanganua kisemwacho ili mawasiliaono yawepo. Methali ni mojawapo wa fasihi ambayo huwa na aina mbili za maana, maana ya wazi na maana fiche. Kwa mfano, 'Kafiri' akufaaye si Islamu asiyekufaa. Kwa kuangalia maana ya juu, 'Kafiri' ni mtu yeyote asiyeamini dini ya Uislamu. Kwa kawaida, waislamu wanapaswa kufaa waislamu wenzao lakini iwapo hatakufaa, sio muislamu tena? Maana fiche ni kwamba, huenda mtu wako wa karibu sana asikufae wakati wa shida fulani lakini ukapata suluhu kutoka kwa mtu ambaye hukutarajia msaada wake.

Nadharia hii inashikilia kuwa maana hutokana na ushirikiano baina ya msikilizaji na msemaji. Hoja hii ina maana kwamba lazima makundi haya mawili yaelewe muktadha na mazingira ya fasihi husika. Hivyo basi, nadharia hii inatusaidia kufahamu kwamba jambo la msingi la chochote kisemwacho ni maana. Iwapo mtu fulani hataelewa hata maana ya msingi ya maneno yanayotumiwa katika methali za Kiswahili, huenda asielewe maana inayoletwa na methali hiyo. Pili, hataweza kuona ukinzani katika methali alizotajiwa wala kuzihusisha methali hizi na mazingira yaliyomo.

Nadharia hii inasisitiza namna ambavyo maana ya maneno katika methali yalivyo muhimu hata kuliko maneno yenyewe. Kwa mfano, iwapo mtu akikupigia methali; "usitukane wakunga, uzazi ungalipo", hii ni methali ambayo maana yake ya ndani ni muhimu sana kuliko maneno yenyewe. Ni methali ambayo inaweza ikatumiwa kwa mtoto \{ambaye hana haja na mkunga\}, wanaume au hata kina mama ambao wana uelewa mkubwa sana wa mkunga na 
umuhimu wake katika jamii.. Neno mkunga ni neno ambalo linabeba maana nyingi. Kwa hivyo anayepigiwa methali hii lazima auhusishe ukunga huu na maisha au mazingira yake halisi na sio tu katika mazingira ya uzazi. Maana ya nje ya neno mkunga, inaweza kuwa mtu yeyote katika maisha yako ambaye ana uwezo wa kukuauni kwa njia moja au nyingine.

\section{Eneo la Utafiti na Uteuzi wa Sampuli}

Eneo lililofanyiwa utafiti ni kata ya Township ambayo iko katika eneo bunge la Kitui ya Kati nchini Kenya. Waliotafitiwa ni wanafunzi wa kidato cha tatu na cha nne kutoka shule za upili katika eneo hili.

Uteuzi wa sampuli: Utafiti ulihusisha shule nane, kati ya shule kumi na tatu za upili katika kata hii. Shule hizi zilizoshirikishwa ziliteuliwa kinasibu.

Wanafunzi walioteuliwa ni wa vidato vya tatu na nne ikizingatiwa kwamba wamezitumia methali kwa muda wa kutosha na wametangamana na fasihi na kuisoma kwa undani. Jumla ya wanafunzi kumi waliteuliwa kimakusudi kutoka kila shule. Ili kumpa kila mwanafunzi nafasi sawa ya kuteuliwa kushiriki katika zoezi hili, njia ya sampuli nasibu ilitumiwa. Wanafunzi hawa walipokwishateuliwa, mtafiti alikutana nao na kuwaongoza katika kujibu maswali ya hojaji.

\section{Mielekeo ya Wanafunzi wa Fasihi Kuhusu Suala la Ukinzani wa Methali za Kiswahili}

Sehemu hii inajumuisha lengo kuu lautafiti huu, ambapo watafiti walilenga kubainisha haswa, milelekeo ya watafitiwa wao kuhusu ubishi unaopatikana katika methali za fasihi ya Kiswahili. Maoni waliyotoa yalidhihirisha wazi mielekeo yao kuhusu suala hili la utafiti.

\section{Chati Inayoonyesha Matokeo ya Wanafunzi Kuhusu Mielekeo yao Kuhusu Methali Kinzani.}

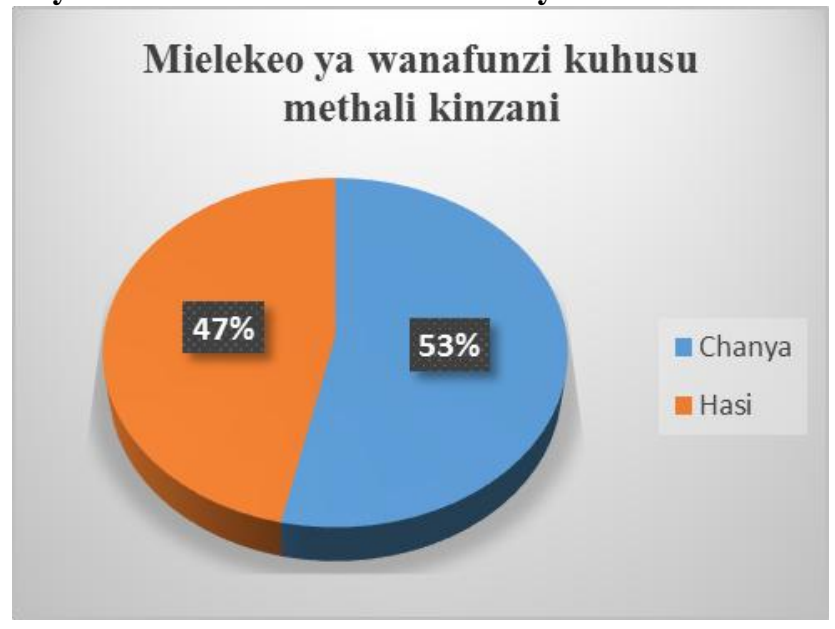

Kwa mujibu wa matokeo ya sehemu hii ya utafiti, ilibainika kwamba asilimia $47 \%$ ya watafitiwa walisema kuwa ukinzani katika methali za Kiswahili huleta mwelekeo hasi huku asilimia 53\% wakisema ukinzani huleta mwelekeo chanya miongoni mwa watumiaji lugha.

Waliosema methali kinzani huleta mwelekeo chanya walitetea msimamo wao kwamba ukinzani katika methali za Kiswahili huleta sura zote za jamii. Kwamba methali hizi hutoa uhalisi wa mambo yanayoathiri maisha ya mwanadamu. Kila methali hutoa maana iliyo na ukweli fulani kutegemea mazingira ya matumizi. Methali kinzani hutoa mwongozo kwa mambo tofauti katika jamii. Kila methali katika jozi ya methali zenye maana kinzani huwa na ujumbe kwa hadhira. Kwa mfano ujumbe wa kujenga urafiki au kuendeleza uadui. Tukiangalia jozi ya methali; adui mpende na Anipendaye nampenda anikataaye nami namkataa, methali ya kwanza inashawishi jamii kuendeleza uhusiano mwema huku ya pili ikipotosha jamii kwamba wa kuenziwa na anayekuenzi.

Watafitiwa wengine walitoa maoni kuwa ukinzani katika fasihi ya Kiswahili ni mojawapo wa nyenzo za kupamba lugha na fasihi. Lugha ya fasihi haipaswi kuwa lugha kavu na kwa hivyo, methali kinzani huchangia katika kuhakikisha kwamba zimeongeza ladha katika lugha hii. Vilevile, watumiaji lugha wanapoendelea kufafanua maana na matumizi ya methali kinzani na hata kutafuta jozi za methali za Kiswahili zilizo na maana kinzani huwa wanakuza ubunifu wao wa kufikiria na zaidi kupevua akili zao. Maoni mengine yaliyotolewa ni kwamba pale 
ambapo kuna methali ambayo ina maana inayopotosha, kuna uwezekano wa kupata methali nyingine ilinayotoa mwelekeo usio wa kupotosha.

Watafitiwa walio na mwelekeo hasi kuhusu methali kinzani walitetea msimamo wao kwa madai kuwa mara nyingi ni vigumu sana kutumia methali kama njia ya kumpa mwanajamii fulani mwongozo kuhusu suala fulani kwani atatumia methali inayotoa maana kinyume kukosoa mawaidha yako. Wengine walisema kuwa methali kinzani hutoa maana inayochanganya na kutatanisha akili za wanajamii. Kwamba methali moja inatoa mwelekeo fulani na nyingine inajitokeza kupinga mwelekeo huo.

Matokeo ya utafiti ni kwamba asilimia kubwa ya watafitiwa ina mwelekeo chanya kuhusu ukinzani katika methali za Kiswahili. Kila methali hubeba maana yake iliyo na ukweli na mwongozo fulani kwa wanajamii. Iwapo jamii itatumia methali katika mazingira mahususi, ukinzani katika methali hautajitokeza. Maoni haya yanaoana na msimamo wa Yankah (1994) kuhusu suala la ukinzani katika lugha.

\section{Hitimisho:-}

Utafiti uliofanywa katika eneo la kata ya township kuhusu ukinzani ulibaini kuwa asilimia kubwa (53\%) ina mwelekeo chanya kuhusu kuwepo kwa ubishi katika methali za Kiswahili. Aidha, asilimia $47 \%$ ya watafitiwa wanamwelekeo ambao kwao, ukinzani unaleta changamoto ya kuelewa maana na matumizi ya methali za Kiswahili. Mtafiti alipiga hatua zaidi ya kutaka kujua iwapo wanajamii wanaona umuhimu wa methali kinzani na matokeo yalibainisha kuwa asilimia $63 \%$ ya watafitiwa wanasema kuwa methali kinzani zina umuhimu huku 37\% wakitoa msimamo kuwa ukinzani katika methali hauna umuhimu wowote. Utafiti huu ulidhirisha wazi kwamba jamii inakumbatia kuwepo kwa ukinzani katika methali za Kiswahili lakini kila methali itumike ipasavyo; kulingana na muktadha.

\section{Marejeleo:-}

1. Abdu, M. (1978) Methali za Kiswahili: Maana na Matumizi Nairobi: Shungwaya Publishers Ltd.

2. B.W Andrzejewski, S. Pilaszewicz, W. Tyloch (1985).Literature in African Languages: Theoretical Issues and Sample Surveys. Cambridge University Press

3. Chinua, A. (1959). Things fall apart. Heinemann Ltd.Nigeria

4. Ergen, Kenneth J. (1990): 212. "Proverbs, pragmatics, and prediction."

5. Felician. (2017). Kuchunguza Ujinsia na Matumizi ya Lugha katika Methali za Wakurya (Doctoral dissertation, The Open University of Tanzania).

6. Grice, H. P. (1975). "Logic and Conversation. "In: Cole, P. \& Morgan, 1. (Eds.), Syntax and Semantics (pp. 4158). New York: Academic Press.

7. John C.N (2011).Dokezo za Sitiari Katika Methali za Kiswahili na Kiganda (Doctoral dissertation. Makerere University).

8. Kalugia L. \& Lodhi A. Y. (1980).Methali Zaidi za Kiswahili Kutoka Afrika Mashariki. Uppsala: Scandinavian Institute of African Studies.

9. Kanu A. (2014). Igbo Proverbs as Embodiments of Igbo-African Philosophy. International Journal of Humanities and Social Science Vol. 4 No.1; pg 164-168

10. King'ei, K. G., \& Ndalu, A. E. (1989). Kamusi ya methali za Kiswahili. East African Publishers.

11. Kipacha, A. (2014). Misemo katika lugha za Magari: Divai mpya? In Swahili Forum (Vol. 21).

12. Kothari, C.R. (2004) Research Methodology: Methods and Techniques (2nd edition.) New Delhi: New Age International (P) Ltd. Publishers

13. Madumulla, J. S.(1995). Proverbs and sayings. Theory and Practice. Dar es Salaam: Institute of Kiswahili Research.

14. Mazrui, A. M \& Syambo, B.K. (1992).Uchambuzi wa Fasihi. Nairobi: East African Educational Publishers.

15. Mhando, P na Balisidya,N. (1976). Fasihi na sanaa za maonyesho. T.P.H. Dar es Salaam

16. Mkota, A. (2009). Kamusi ya methali: maana na matumizi. Vide-Muwa Publishers.

17. Mochiwa, Z. (1988). Mvumilivu hula mbovu. Dar es Salaam University Press.

18. Mtesigwa, G. K. (2013). Kuchunguza Matumizi ya Methali katika Jamii ya Wanyiramba (Doctoral dissertation, The Open University of Tanzania).

19. Mugenda O.M na Mugenda A.G. (2012). Research Methods Dictionary. Nairobi: Applied Research \& Training services.

20. Mulokozi, M.M. (1989). Tanzu za Fasihi Simulizi. Dar es Salaam: TUKI. 
21. Murithi, M.M. (2015). Uchanganuzi wa Methali za Kiswahili: Mtazamo wa Kiudenguzi (Doctoral dissertation, Kenyatta University).

22. Ngole, S. A \& Honero, L.N. (2004). Fasihi - Simulizi: Methali. Taasisi ya Uchunguzi wa Kiswahili: Chuo Kikuu cha Dar es Salaam

23. Njogu, K. \& Chimerah, R. (1999) Ufundishaji wa Fasihi: Nadharia na Mbinu. Nairobi: Jomo Kenyatta foundation

24. Sito, P. S. (2015). Athari za mazingira katika uteuzi wa msamiati: mfano wa methali za Kiswahili zinazorejelea viumbe wa majini (Doctoral dissertation, University of Nairobi).

25. Taylor, R. (1981). Understanding the Elements of Literature. London: Macmillan

26. TUKI (2013). Kamusi ya Kiswahili sanifu. Oxford University Press: East Africa Ltd. Kenya

27. Vasquez, A.S. (1973). Art and Society. New York: Monthly Review Press.

28. Wamitila, K.W. (2001). Kamusi ya methali. Lulu za Lugha 2. Nairobi Longhorn Publishers. Vol. 2.

29. Wamitila, K.W. (2003). Kichocheo cha Fasihi Simulizi na Andishi. English Press, Nairobi.

30. Wamitila, K. W. (2008). Misingi ya uchanganuzi wa fasihi (Vol. 1). Vide-Muwa Publishers.

31. Wellek, R, W. (1949). Theory of Literature. New York: Harvest.

32. Yankah, K. (1989). The Proverb in the Context of Akan Rhetoric. New yolk: Peter Lang Publishing

33. Yankah, K. (1994). Do proverbs contradict? Wise Words, 127-41.New yolk: Peter Lang Publishing 